\title{
The Effect of BAFF Inhibition on Autoreactive B-Cell Selection in Murine Systemic Lupus Erythematosus
}

\author{
Alexis Boneparth, ${ }^{1 *}$ Megan Woods, ${ }^{1 *}$ Weiqing Huang, ${ }^{1}$ Meredith Akerman, ${ }^{2}$ Martin Lesser, ${ }^{2}$ and Anne Davidson ${ }^{1}$ \\ ${ }^{1}$ Center for Autoimmunity, Feinstein Institute for Medical Research, Manhasset, New York, United States of America; \\ and ${ }^{2}$ Musculoskeletal Diseases and Biostatistics Unit, Feinstein Institute for Medical Research, Manhasset, New York, \\ United States of America
}

\begin{abstract}
The goal of this study was to determine how B-cell-activating factor of the TNF family (BAFF) availability influences selection of the autoreactive B-cell repertoire in NZB/W and NZW/BXSB lupus-prone mice bearing the site-directed heavy-chain transgene $3 \mathrm{H} 9$ that encodes for anti-dsDNA and anti-cardiolipin $(\mathrm{CL})$ autoantibodies. We used a bone marrow chimera system in which autoreactive $3 \mathrm{H} 9$ transgenic B cells were allowed to mature in competition with wild-type cells and could be identified by green fluorescent protein. The light-chain repertoire associated with the $3 \mathrm{H} 9$ heavy chain in naive and antigen-activated B-cell subsets was assessed using single-cell polymerase chain reaction. We found that deletion of autoreactive transgenic B cells occurred in the bone marrow of both strains regardless of BAFF availability, and there were only modest and physiologically non-relevant effects on the naive B-cell repertoire. BAFF inhibition had different effects on selection of the germinal center repertoire in the two strains. In the NZW/BXSB strain, BAFF inhibition phenocopied the loss of one TLR7 allele in that it influenced the selection of $3 \mathrm{H} 9$-encoded autoreactive B cells in the germinal center but did not prevent somatic mutation. In the NZB/W strain, BAFF inhibition did not alter the selection of $3 \mathrm{H} 9$-encoded B cells in the germinal center, but it influenced selection of a subset of germinal center cells into the plasma cell compartment. Our data underscore the complexity of regulation of the autoreactive B-cell repertoire by BAFF and may help to explain the heterogeneity of responses observed after BAFF inhibition in humans.
\end{abstract}

Online address: http://www.molmed.org

doi: 10.2119/molmed.2016.00022

\section{INTRODUCTION}

Systemic lupus erythematosus (SLE) is an autoimmune disorder in which loss of tolerance to nucleic acids and their binding proteins leads to the production of autoantibodies that cause systemic inflammation and organ damage. Both intrinsic and extrinsic defects in B-cell tolerance can contribute to the generation of autoantibodies in SLE (1). Naive autoreactive B cells may expand in extrafollicular regions or may undergo clonal expansion in germinal centers (GCs) (2-5). B cells that acquire autoreactivity de novo as a consequence of somatic mutation also contribute to the autoreactive repertoire $(3,6,7)$. Somatic mutation requires help from T cells, but in the setting of inflammation, it may occur outside GCs as well as within GCs $(2,8)$. Finally, innate signals may enhance autoreactive responses in a $\mathrm{T}$ cell-independent fashion (9). Understanding how these defects contribute to autoantibody production in individual patients will enable therapy to be directed to the appropriate B-cell stage and subset.

*AB and MW contributed equally to this work.

Address correspondence to Anne Davidson, Feinstein Institute for Medical Research, 350 Community Drive, Manhasset, NY 11030. Phone: 516-562-3840; Fax: 516-562-2953;

E-mail: adavidson1@nshs.edu.

Submitted January 20, 2016; Accepted for publication February 8, 2016; Published Online (www.molmed.org) February 12, 2016.

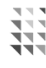

Feinstein Institute

for Medical Research

Northwell Health

B cell-activating factor of the TNF family (BAFF) is a cytokine that acts as a homeostatic regulator of B-cell selection and survival. High levels of BAFF are associated with autoimmunity, particularly SLE. Belimumab, a monoclonal anti-BAFF antibody, has recently been approved for the treatment of SLE $(10,11)$. BAFF and its receptors play multiple roles in B-cell development (12-14). During the transitional stage, signals through the BAFF receptor (BAFF-R) and the BCR cooperate in several ways to enhance B-cell survival. Transitional autoreactive B cells often downregulate their BCR and therefore require a higher amount of signal through BAFF-R to survive. If BAFF levels are limiting, these cells may be deleted or may be directed to the marginal zone, but if BAFF levels are high, they are more likely to survive (rev. in 14). Autoreactive $B$ cells that escape into the naive repertoire under the influence of high circulating levels of BAFF could give rise to pathogenic autoantibodies if they undergo extrafollicular clonal expansion or if they 
are permitted to enter and expand in the GC. BAFF signaling, especially through its receptor transmembrane activator and calcium modulator ligand interactor, belonging to the tumor necrosis factor (TNF) receptor family (TACI), also interacts with the MyD88 pathway and can amplify innate immune signals received through nucleic acid sensing toll-like receptors (15). This step further promotes autoreactive B-cell activation and expansion.

The GC reaction produces IgG autoantibodies that can improve their affinity for autoantigens or that acquire autoreactivity de novo as a result of somatic mutation. Optimal GC maintenance requires BAFF, since both $B A F F$ and $B A F F-R \mathrm{KO}$ mice form smaller GCs that decline prematurely (16). BAFF is required for the development of a mature follicular dendritic cell (FDC) reticulum (16) and also influences inducible T-cell costimulator ligand (ICOSL) expression on B cells, thus regulating the ability of B cells to promote follicular $\mathrm{T}$ helper cell (TFH) expansion during GC development (17). TFH, in turn, produces BAFF, and this may enhance the survival and selection of high-affinity B cells (18). Furthermore, activated T cells are also responsive to BAFF, releasing interferon- $\gamma$ that amplifies autoimmune effector responses (19). The ability of BAFF inhibition to regulate plasma cells that arise either from naive autoreactive B cells or from the GC has not been fully explored in spontaneous models of SLE.

The purpose of the experiments reported here was to determine how BAFF availability influences the selection of naive and antigen-selected autoreactive B cells bearing the site-directed anti-cardiolipin (CL)/DNA immunoglobulin heavy-chain $\mathrm{V}$ region transgene $3 \mathrm{H} 9$ during the evolution of SLE in NZW/ $\mathrm{BXSB}$ and NZB/W mice that express anti-CL and anti-dsDNA autoantibodies, respectively. The use of $3 \mathrm{H} 9$ bone marrow chimeras allowed us to study selection of the 3H9-encoded B-cell repertoire in the setting of both physiologic competition with non-autoreactive B cells and evolving inflammation. Because high affinity anti-dsDNA and anti-CL autoreactivity in the $3 \mathrm{H} 9$ system is acquired by somatic mutation (7), we could analyze the effects of BAFF inhibition on both the naive repertoire and on the de novo generation of high-affinity autoreactive $B$ cells. We show that the effects of BAFF inhibition are variable, depending both on strain and on individual Ig genes.

\section{MATERIALS AND METHODS}

\section{Mice}

$\mathrm{GFP}^{+}$3H9 NZW mice were generated as previously reported (20). 3H9.GFP ${ }^{+}$. NZW heterozygous females were bred with BXSB males, and 3H9.GFP ${ }^{+}$NZW males were bred with NZB females to produce $\mathrm{GFP}^{+}$male NZW /BXSB F1 or female $\mathrm{GFP}^{+} \mathrm{NZB} / \mathrm{W}$ F1 progeny with or without the $3 \mathrm{H} 9$ transgene (Supplementary Table S1). Wild-type NZW / BXSB mice were made by crossing the parental NZW and BXSB strains. BXSB males, NZB females and wild-type NZB/W females were purchased from The Jackson Laboratory.

Bone marrow chimeras were generated by transfer of $50 \% 3 \mathrm{H} 9 . \mathrm{GFP}^{+}$and $50 \%$ wild-type bone marrow from either NZW/BXSB males or NZB/W females into totally irradiated 8- to 12 -wk-old recipients of the same strain and sex. Groups of control wild-type chimeras were made using $\mathrm{GFP}^{+}$non-3H9 donors. Treatment with BAFF-R-Ig (21) 500 mg intraperitoneally three times per week was begun in groups of 3H9.NZW/BXSB and 3H9.NZB/W chimeras $3 \mathrm{~d}$ after bone marrow transplant and continued for the duration of the experiment. Controls were left untreated. Chimeric mice were tested for proteinuria every 2 wks (Multistick; Fisher) and bled periodically for serologic analyses $(22,23)$. Mice were killed for analysis at the onset of established proteinuria of $300 \mathrm{mg} / \mathrm{dL}$ on at least two occasions $1 \mathrm{wk}$ apart (Supplementary Table S1). Studies were approved by the IACUC of the Feinstein Institute for Medical Research (Protocols 2009-054 and 2007-038).

\section{Quantitation of Autoantibodies to Cardiolipin and dsDNA}

Serial enzyme-linked immunosorbent assays (ELISAs) of serum for antibodies to $\mathrm{CL}$ and dsDNA were performed as previously described $(22,24,25)$.

\section{Flow Cytometry and Sorting}

Spleen and bone marrow B cells were analyzed as previously described $(21,26,27)$. For each stain, the FITC channel was left open to enumerate green fluorescent protein- positive $\left(\mathrm{GFP}^{+}\right)$cells.

\section{Single-Cell Sorting and Polymerase Chain Reaction}

$\mathrm{GFP}^{+}$single cells were sorted from follicular and GC B-cell and plasma cell subsets from four to six mice per group. cDNA preparation and polymerase chain reaction (PCR) of the $3 \mathrm{H} 9$ heavy

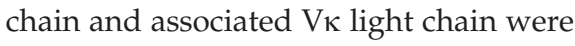
performed as previously described, using a constant region IgM primer for naive cells and a constant region IgG primer for GC and plasma cells $(7,28)$. PCR products were sequenced by Genewiz, and sequences were identified using the International ImMunoGeneTics (IMGT) database. To distinguish between $V \kappa 5-43^{*} 01$ and $V \kappa 5-45^{*} 01$ that differ by three base pairs at the $5^{\prime}$ end and to enumerate somatic mutations, $5^{\prime}$ leader primers for $V \kappa 5-43 / 45^{*} 01$ and $V \kappa 5-48^{*} 01$ were used to generate full-length lightchain genes for resequencing (20). Somatic mutation rates were calculated by using a program available on the IMGT website.

\section{Statistics}

Proteinuria and survival data in Figure 1 were analyzed by using Kaplan-Meier curves and log-rank test. Comparisons shown in Figures 1A, 1B, 2 and 3 were performed by using the Mann-Whitney $U$ test. The $p$ values $\leq 0.05$ were considered significant. Comparisons in Figure 4 were performed using a pair-wise Mann-Whitney $U$ test with a Bonferroni adjustment to account for multiple testing. To preserve an overall 5\% significance level, a pairwise comparison 
was considered statistically significant at $p<0.025$. Statistical analysis of the Vк repertoire data shown in Figures 5 and 6 was performed as previously described (3). For additional details on the statistical methodology, please refer to Lesser et al. (29). Comparisons in Figure 7 were performed using $\chi^{2}$ analysis.

All supplemental materials are available online at www.molmed.org.

\section{RESULTS}

\section{Clinical Phenotype of 3H9 W/B Bone} Marrow Chimeras

Male NZW/BXSB (M W/B wild-type) and 3H9 transgenic (M W/B 3H9) chimeric mice (Supplementary Table S1) developed IgG autoantibodies to CL by $10-13$ wks posttransplantation. BAFF-R-Ig-treated NZW / BXSB 3H9 (M W/B 3H9 BAFF-R-Ig) mice developed anti-CL antibodies with the same kinetics as M W/B 3H9 mice but had significantly lower maximal titers at $16-18$ wks $(p<0.05$, Figure $1 \mathrm{~A})$. BAFF-R-Ig-treated female NZB/W mice (F B/W 3H9 BAFF-R-Ig) developed high titer anti-dsDNA antibodies by $18 \mathrm{wks}$ posttransplantation that were no different from untreated female 3H9 NZB/W mice (F B/W 3H9, Figure 1B) $(21,22)$. Proteinuria onset was delayed by BAFF-R-Ig treatment in chimeras of both strains (Figured 1C, D), as has been observed in the wild-type strains $(21,22)$.

\section{B-Cell Phenotype of 3H9 Bone Marrow Chimeric Mice}

The spleens of BAFF-R-Ig-treated 3H9 mice were significantly smaller than those of their untreated counterparts (M 3H9 W / B BAFF-R-Ig, $74.3 \pm 38.5$ versus $137.7 \pm 69.4 \times 10^{7}$ cells per spleen; $p<0.05$; F 3H9 B/W BAFF-R-Ig $55.3 \pm$ 22.5 versus $235.0 \pm 160.7 \times 10^{7}$ cells per spleen; $p<0.005)$. As expected, phenotypic analysis of spleen B cells indicated that BAFF-R-Ig-treated mice had significant depletion of B cells compared with untreated $3 \mathrm{H} 9$ chimeras with sparing of the T1, GC and plasma cell subsets in both strains (Figure 2). The total number of CD4 and CD8 T cells per spleen was not altered by BAFF-R-Ig treatment in either strain (data not shown).
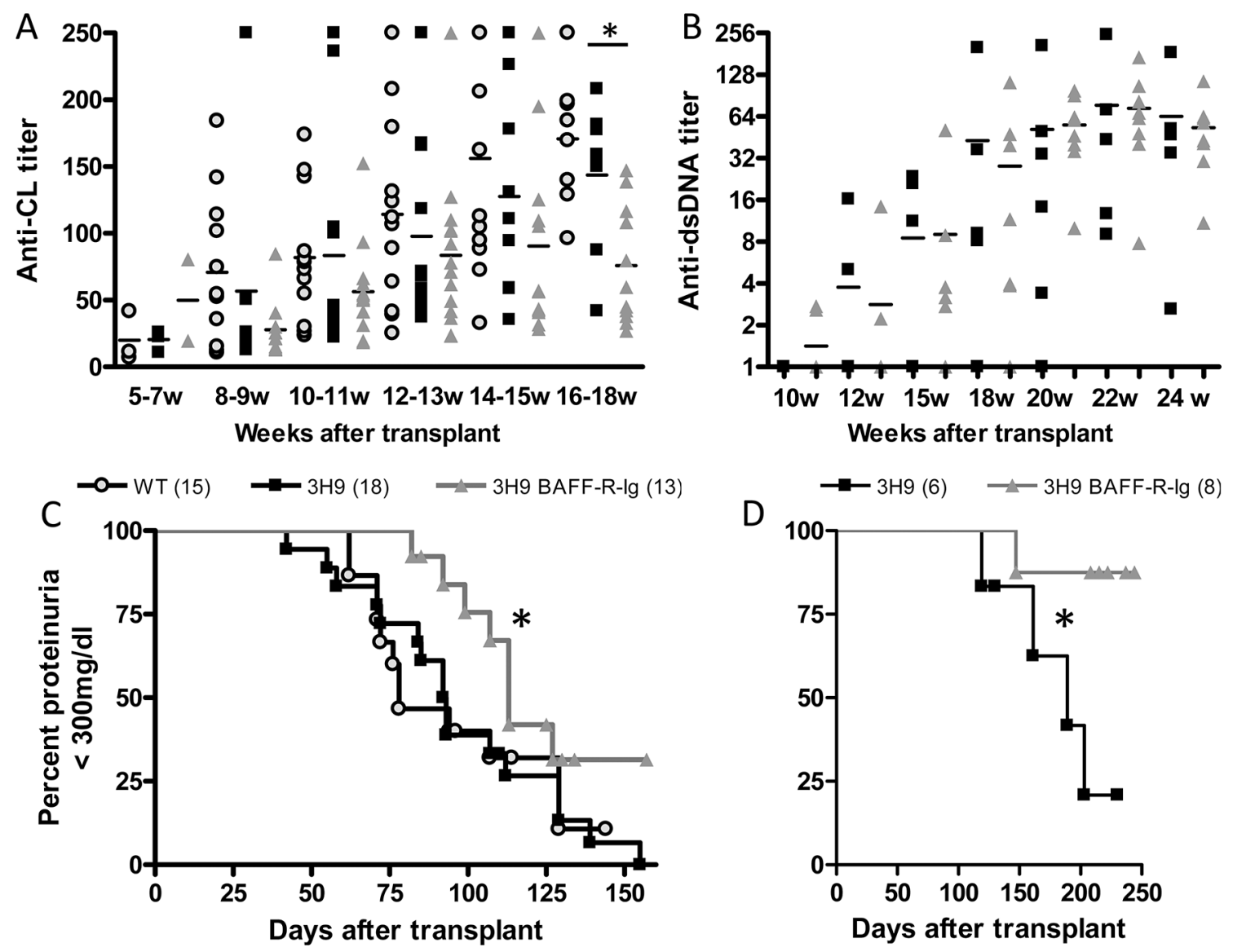

Figure 1. Clinical outcomes of BAFF-R-Ig-treated 3H9 chimeric mice. (A, C) Male NZW/BXSB mice. (B, D) Female NZB/W mice. (A, B) ELISA values for each antigen were normalized to a high titer control run on each plate and are shown for each individual mouse at the indicated times after bone marrow transplant. The horizontal bar indicates the mean for each group $\left({ }^{*} p<0.05\right)$. (C, D) Kaplan-Meier plots show significant delay in the onset of fixed $300 \mathrm{mg} / \mathrm{dL}$ proteinuria in BAFF-R-Ig-treated mice of both strains $\left({ }^{*} p<0.05\right.$ versus untreated mice). 

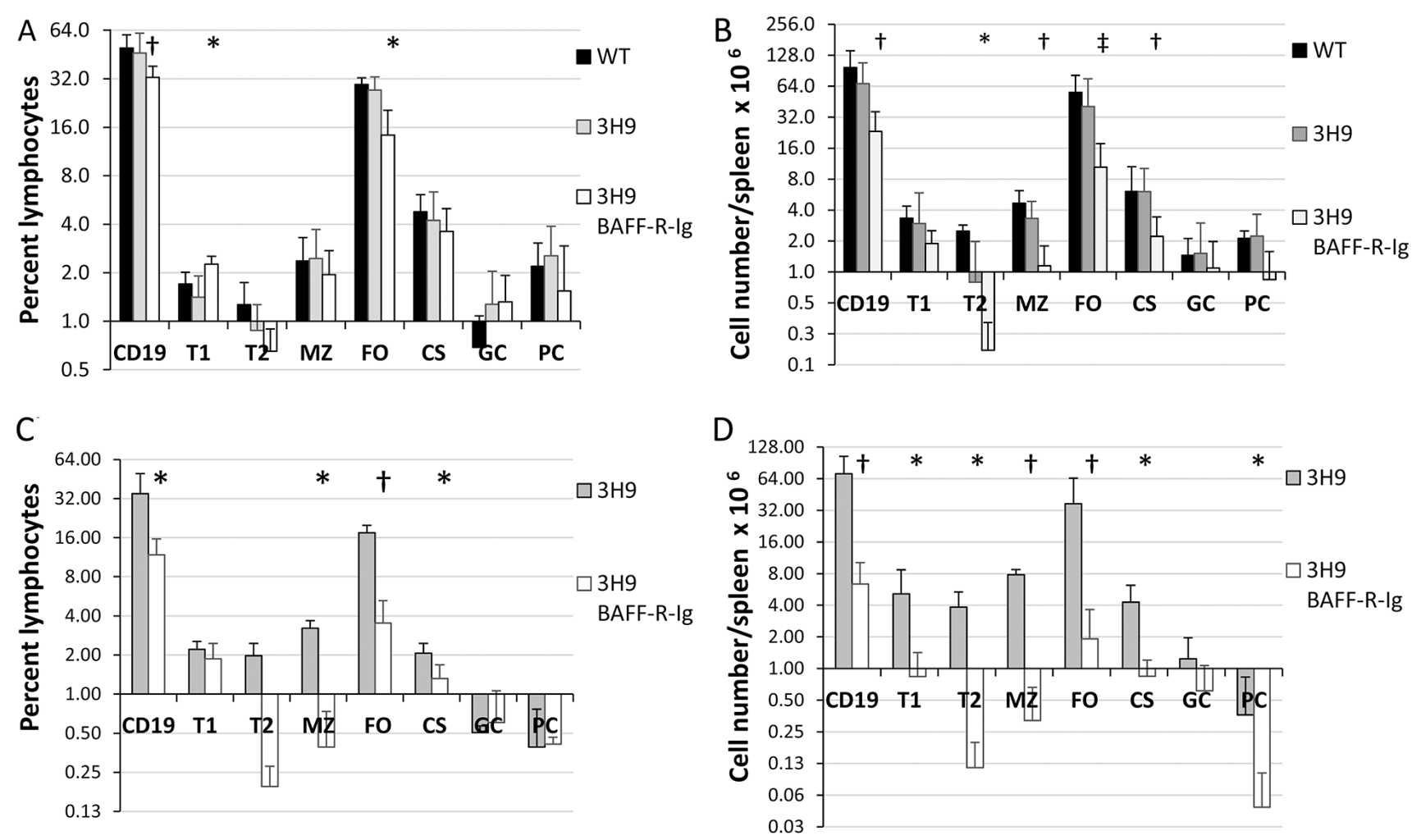

Figure 2. BAFF-R-Ig treatment depletes naive B cells. (A, C) Frequency of each B-cell subset as a percentage of total lymphocytes in NZW/BXSB male (A) and NZB/W female (B) chimeras. Four to eight mice were examined per group $\left({ }^{*} p<0.05 ; \dagger p<0.01 ;{ }^{\ddagger} p<0.001\right)$. $(B, D)$ Total number of $B$ cells in each subset per spleen. $n=4-8$ per group; ${ }^{*} p<0.05 ;{ }^{\dagger} p<0.01 ;{ }^{\dagger} p<0.001,3 \mathrm{H} 9$ untreated versus 3H9 BAFF-R-Ig-treated chimeras (T1, T2, transitional type 1 and 2 B cells; MZ, marginal zone; FO, follicular; CS, class switched; PC, plasma cell).

As a measure of the selection pressure against transgenic B cells, we assessed deletion of $3 \mathrm{H} 9 \mathrm{~B}$ cells in each subset by calculating the ratio of $\% \mathrm{GFP}^{+} \mathrm{B}$ cells to $\% \mathrm{GFP}^{+}$non-B cells (non-B cells for BM and average of spleen CD4 and CD8 cells for spleens) (20). In the absence of deletion, this ratio should be 1 . Ratios of $\%$ $\mathrm{GFP}^{+} \mathrm{CD} 4$ cells to $\% \mathrm{GFP}^{+} \mathrm{CD} 8$ were close to 1 for all chimeras (data not shown), indicating no selection pressure against $\mathrm{GFP}^{+} \mathrm{T}$ cells. As we have previously reported in NZW/BXSB mice (20), the untreated $3 \mathrm{H} 9$ chimeras exhibited deletion of $\mathrm{GFP}^{+} / 3 \mathrm{H}^{+}$transgenic B cells in the bone marrow that occurred as early as the pre-B-cell stage with a modest amount of further deletion occurring between the pre-B-cell and transitional B-cell stages and no further deletion between the transitional and follicular stages (Figures 3A, C). A similar deletion pattern was found in the NZB/W strain (Figures 3B, D). Treatment with BAFF-R-Ig had no additional effect on deletion of transgenic B cells at any of these B-cell stages in either strain (Figures 3A-D).

Surface IgM staining was decreased on splenic $\mathrm{GFP}^{+}$follicular cells from $3 \mathrm{H}^{+}$chimeric mice compared with $\mathrm{GFP}^{-}$ follicular B cells from the same mice, suggesting that the $3 \mathrm{H}^{+}$cells have encountered autoantigen in the periphery $(30,31)$. This BCR downregulation was not altered by BAFF-R-Ig treatment in either strain (Figure $3 \mathrm{E}$ ). $\mathrm{GFP}^{+}$transgenic $B$ cells were preferentially enriched in the GCs of both strains of mice, indicating expansion of the autoreactive transgenic population; this was not altered by BAFF-R-Ig treatment in either strain (Figure 3F).

\section{Single Cell Analysis of the 3H9-Associated Light-Chain Repertoire of NZB/W Mice}

We previously established that reconstituted unmutated $3 \mathrm{H} 9 / \mathrm{V \kappa}$ combinations using $V \kappa 1-117,3-4,3-12$, 4-55, 9-120, 13-85 and 16-104 are nonreactive with dsDNA, CL, histones or chromatin, whereas those using Vк1-110, 4-57, 4-57*1-01, 4-79, 5-43, 5-45, 5-48, 6-15, 8-30, 9-123, 10-94 and $12-46$ react with one or more of these antigens (7; data not shown). In 3H9 NZW / BXSB mice, we further reported that the GC repertoire is dominated by members of the $\mathrm{V} \kappa 5$ family, particularly $V \kappa 5-43 / 5$ and $V \kappa 5-48$ that are much less commonly expressed in the follicular or marginal zone repertoires (20). In this study, we likewise examined the repertoire of naive 

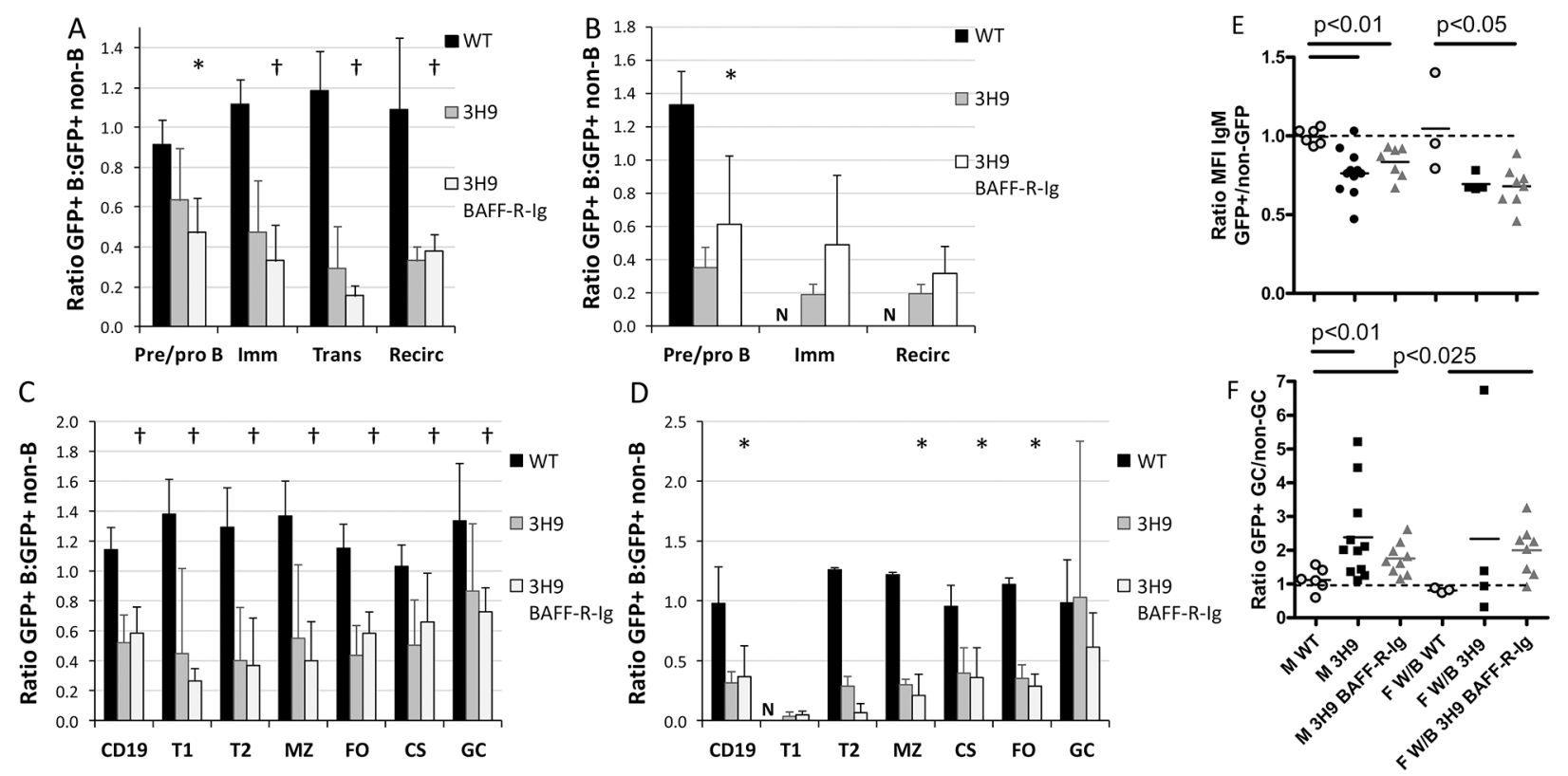

Figure 3. Deletion of $3 \mathrm{H} 9 \mathrm{~B}$ cells occurs in the bone marrow and is not further affected by BAFF-R-Ig. (A, B) Ratio of GFP' B cells to GFP ${ }^{+}$ non-B cells in bone marrow subsets shows deletion occurring at the pre-B cell stage in the $3 \mathrm{H} 9$ chimeras. Further deletion occurred between pre-B cells and recirculating B cells (Imm, Immature; Trans, transitional; Recirc, recirculating). (C, D) Ratio of GFP' B cells: GFP' $T$ cells in spleens shows no further deletion after the Tl stage and no difference between $3 \mathrm{H} 9$ untreated and BAFF-R-lg-treated chimeras. $n=4-8$ per group; symbols show comparisons of ratios of BAFF-R-Ig-treated mice with those of wild-type counterparts: * $p<0.05$, ${ }^{\dagger} p<0.01$, compared with wild-type chimeras. (E) Downregulation of slgM as assessed by the ratio of slgM on GFP" follicular B cells: slgM on GFP' follicular B cells occurs in all the $3 \mathrm{H} 9$ chimeras and is not altered by BAFF-R-lg treatment. (F) Overrepresentation of $3 \mathrm{H} 9 \mathrm{~B}$ cells among GC B cells in $3 \mathrm{H} 9$ chimeras, as assessed by the ratio of GFP+ $G C$ cells: GFP- GC cells is not influenced by BAFF-R-lg treatment.

B-cell subsets and class switched B cells from young and old $3 \mathrm{H} 9 \mathrm{NZB} / \mathrm{W}$ mice that were the donors for the NZB/W chimeras. As we have shown in the NZW / BXSB strain, the transitional, marginal zone and follicular repertoires were different from each other (Supplementary Figure S1A; data not shown). The marginal zone repertoire was particularly restricted in both young and aged mice, with almost $50 \%$ of the repertoire accounted for by two

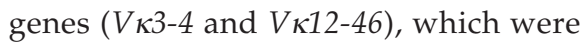
less commonly expressed in the follicular repertoire; $V \kappa 12-46$ is also highly expressed by marginal zone $B$ cells of the NZW/BXSB strain. By contrast, the GC and class switched repertoires of aged 3H9 NZB/W mice were dominated by a restricted set of genes that were uncommon in the follicular and marginal zone repertoires, including

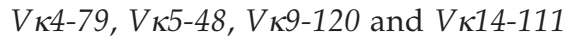

(Supplementary Figure 1B). Of these, $V \kappa 5-48$ and $V \kappa 9-120$ are also expressed by GC B cells of NZW / BXSB mice.

\section{Single-Cell Analysis of the Naive 3H9-Associated Light-Chain Repertoire of Chimeric Mice}

To determine whether BAFF-R-Ig treatment altered selection of the naive B-cell repertoire, we performed single-cell PCR of light chains associated with the 3H9 heavy chain in the mature follicular B-cell subset from BAFF-R-Ig-treated and -untreated chimeric mice (Supplementary Table S2). Analysis of the follicular light-chain repertoire in chimeras of the $\mathrm{NZB} / \mathrm{W}$ strain revealed that treatment with BAFF-R-Ig decreased the frequency of $V \kappa 3-4$, a light chain that is relatively confined to the marginal zone in the parental 3H9 donors, and increased the frequency of $V \kappa 3-1$ and $V \kappa 13-84 / 85$. However, there was no clear effect on selection of those Vк genes known to be autoreactive versus those with no known SLE-related autoreactive specificity. Notably, the autoreactive light-chain genes $V \kappa 5-43 / 5$ and $V \kappa 5-48$ were present at measurable frequency in the follicular repertoire of NZB/W chimeras, but their representation was no different in BAFF-R-Ig-treated mice than in controls (Figure 4A, Supplementary Table S2). In NZW/BXSB mice, there were only minor differences between the follicular repertoires of BAFF-R-Ig-treated mice and control mice. Of these, only a decrease in $V \kappa 3-4$ was shared with the NZB/W strain (Figure 4B, Supplementary Table S2).

\section{Single-Cell Analysis of the GC and Plasma Cell 3H9-Associated Light- Chain Repertoire of Chimeric Mice}

The 3H9-associated repertoire of light chains in class-switched GC B cells and plasma cells is highly restricted in male 


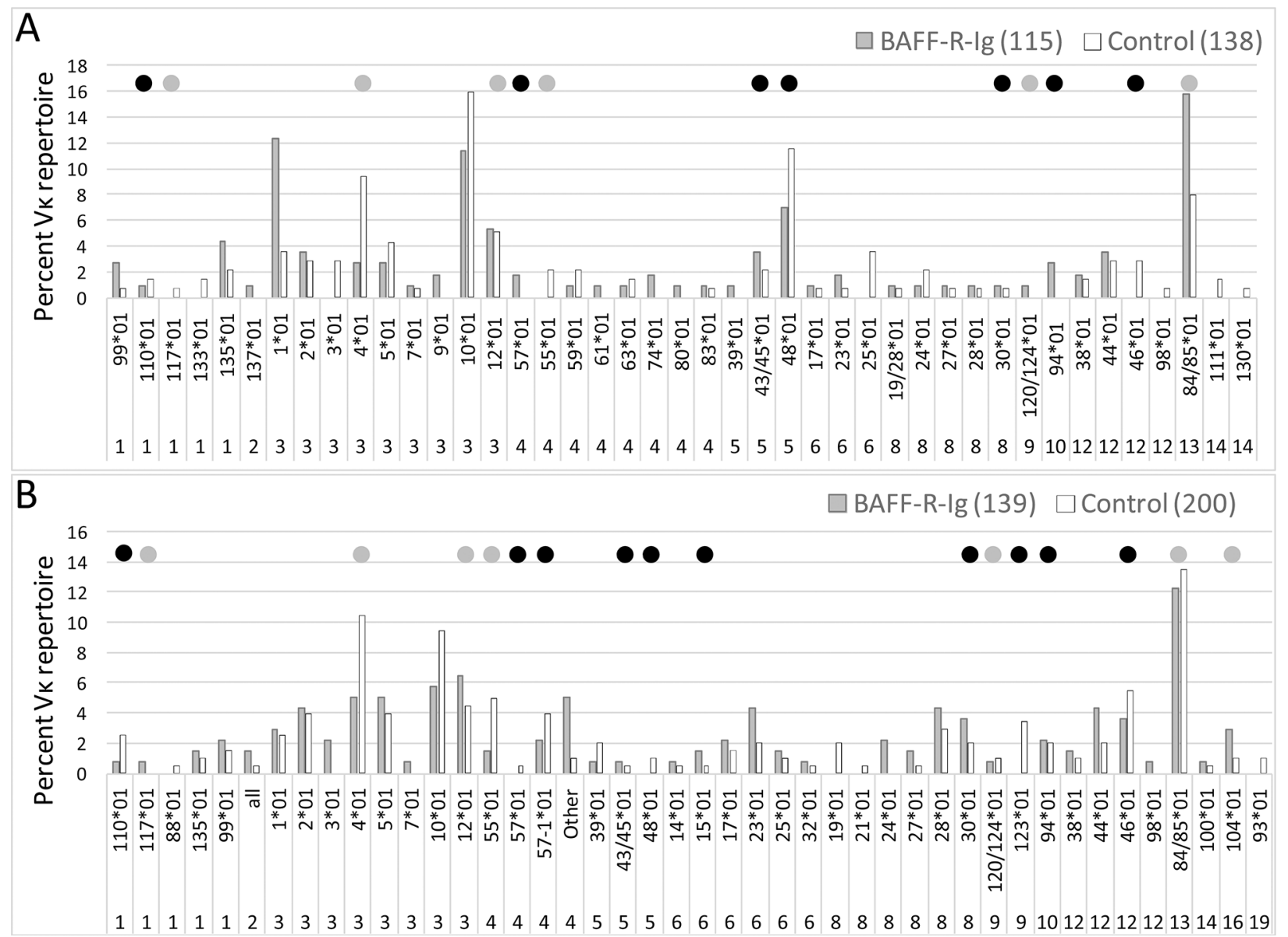

Figure 4. The repertoire of $V_{\kappa}$ light chains associated with the $3 \mathrm{H} 9$ heavy chain in follicular B-cell subsets from NZB/W (A) and NZW/BXSB (B) chimeras. The $x$ axis shows the light-chain gene family and specific genes that were expressed. The $y$ axis shows percent of the total repertoire for each subset. Four to six spleens were analyzed for each subset (see Supplementary Tables S2 and S3 for raw data and statistical analysis). Black dots indicate $V_{\kappa}$ genes that confer specificity for dsDNA, histones or CL. Grey dots indicate $V_{\kappa}$ genes without these specificities.

NZW /BXSB mice with overrepresentation of $V \kappa 5-43 / 45$ and $V \kappa 5-48$ that confer specificity for chromatin and other autoantigens including dsDNA, CL and histone (7). In the NZW/BXSB chimeras, treatment with BAFF-R-Ig resulted in decreased expression of $V \kappa 5-48$ relative to $V \kappa 5-43 / 45$ in the GC repertoire (Figure 5A); this change was also observed in the plasma cell repertoire (Supplementary Tables S2 and S3). Despite a difference in the autoantibody profile between NZW/BXSB and NZB/W mice, $V \kappa 5$ light chains were also overrepresented in female NZB/W chimeras, reflecting the ability of 3H9/Vк5-encoded autoantibodies to acquire either high-affinity dsDNA or high-affinity CL specificity by somatic mutation (7). No differences were observed in the frequency of $\mathrm{V} \kappa 5$-encoded light chains in the GCs of BAFF-R-Ig-treated and control NZB/W chimeras (Figure 5B, Supplementary Tables S2 and S3).

We previously showed that the autoreactive specificity of germline encoded $3 \mathrm{H} 9 / \mathrm{V} \kappa 5$ antibodies in non-chimeric $3 \mathrm{H} 9 \mathrm{~W} / \mathrm{B}$ males and females is influenced by the associated $\mathrm{J} \kappa(7)$. In partic-

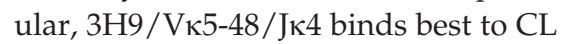
and dsDNA, whereas 3H9/Vк5-43/Jк5 binds to histone and dsDNA. In the NZW/BXSB chimeras, treatment with BAFF-R-Ig resulted in an increase in the representation of $3 H 9 / V \kappa 5-43 / J \kappa 5$ and a decrease in the representation of $V \kappa 5-48 / J \kappa 4$ in the GC repertoire (Figure 6A, $p<0.001$ ); a similar difference was also present in the plasma cell repertoire (data not shown). However, no such differences were observed in the GC repertoire of $\mathrm{NZB} / \mathrm{W}$ chimeras (Figure 6B).

$3 \mathrm{H} 9 / \mathrm{V} \kappa 5-45 / \mathrm{J} \kappa 5$ binds with higher affinity to dsDNA, CL and histones than does $3 \mathrm{H} 9 / \mathrm{V} \kappa 5-43 / \mathrm{J} \kappa 5$. Because the sequencing method we used does not distinguish between $V \kappa 5-43$ and $V \kappa 5-45$, which have only two amino acid differences in the $5^{\prime}$ region ( $S$ to $R$ in FR1 and $\mathrm{N}$ to $\mathrm{Y}$ in $\mathrm{CDR} 1$ ), we performed 


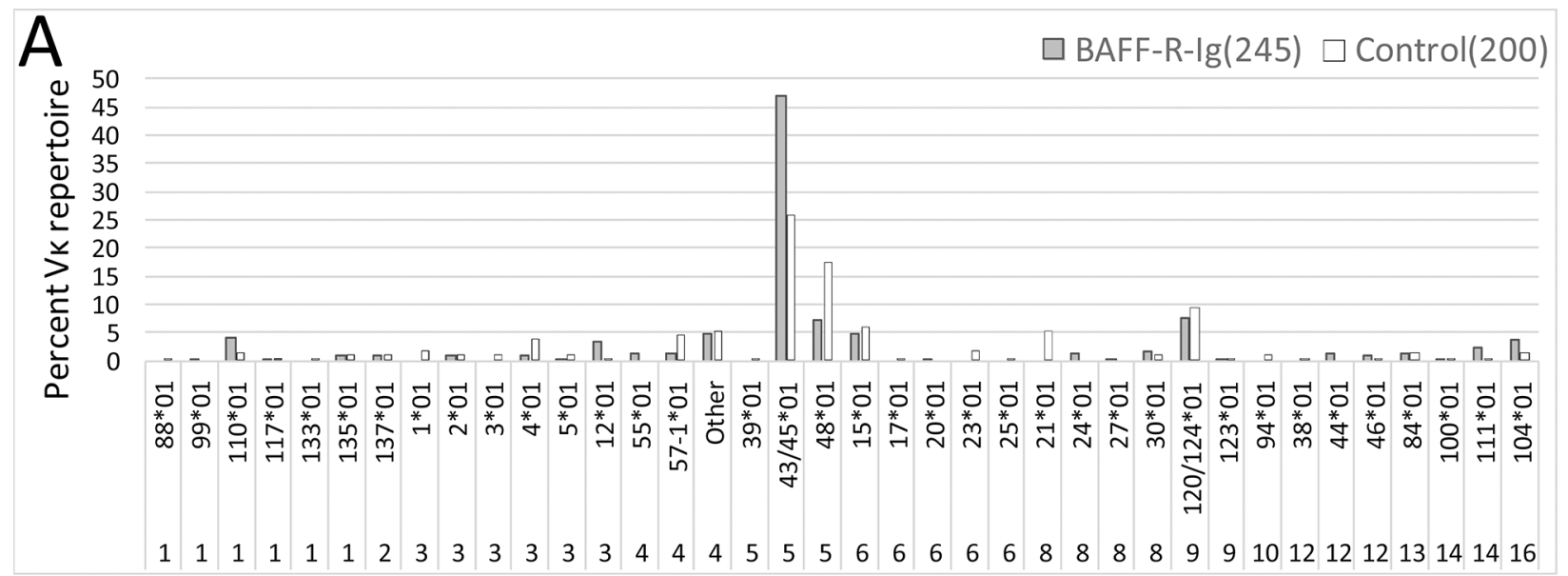

B

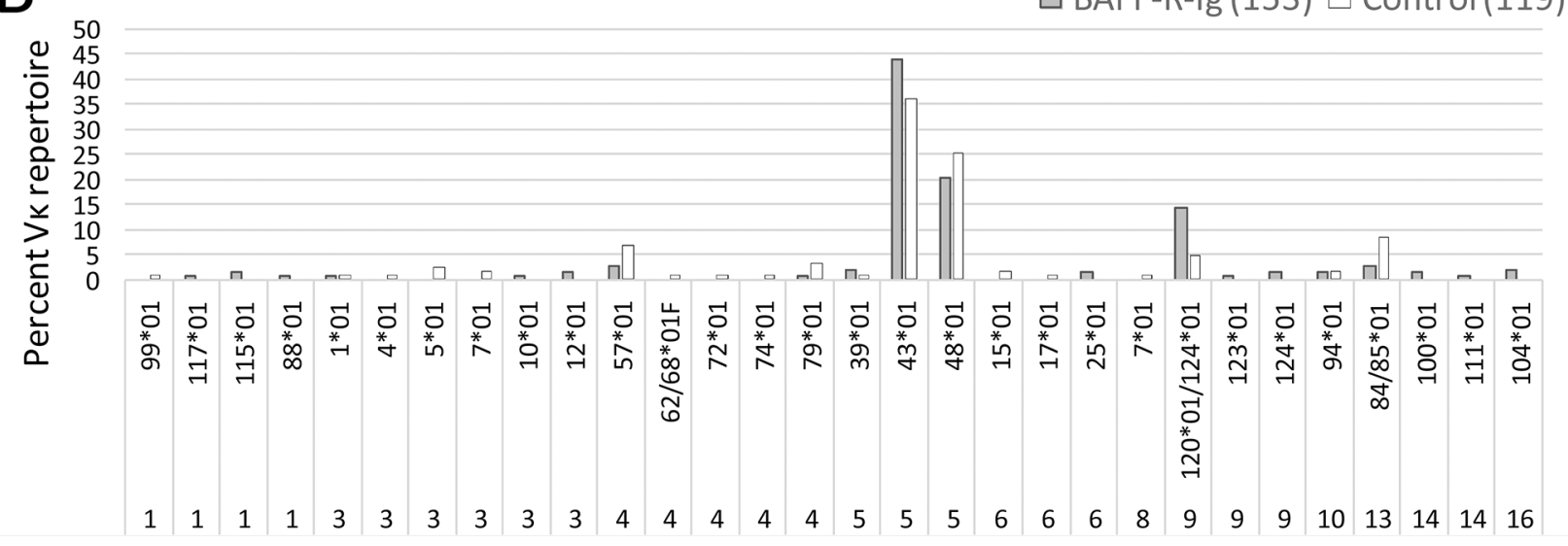

Figure 5. The repertoire of $V_{\kappa}$ light chains associated with the $3 \mathrm{H} 9$ heavy chain in GC B-cell subsets from NZW/BXSB (A) and NZB/W (B) chimeras. The $y$ axis shows percent of the total repertoire for each subset. Four to six spleens were analyzed for each subset (see Supplementary Tables S2 and S3 for raw data and statistical analysis).

full-length sequencing of the light chains from the chimeras. We found that $V \kappa 5-45$ constituted $23.7 \%$ of the $V \kappa 5-43 / 45$ light chains in the GCs of control 3H9 NZW/ BXSB chimeras compared with $17.5 \%$ of BAFF-R-Ig-treated chimeras; this difference was not significantly different. Similarly, $V \kappa 5-45$ was represented equivalently in the plasma cell repertoire of BAFF-R-Ig-treated and untreated NZW / BXSB chimeras. In NZB/W mice, $V \kappa 5-43$ was the dominant light chain expressed in the GCs, constituting $89 \%$ and $100 \%$ of $V \kappa 5-43 / 45$ light chains in control and BAFF-R-Ig-treated mice, respectively.

We have previously shown by analysis of hybridomas that high-affinity $\mathrm{CL}$ and dsDNA binding activity of

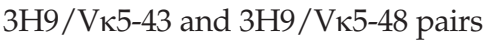
can be acquired as a result of somatic mutations (7). Because $3 H 9 / V \kappa 5-48$ was not commonly found in BAFF-R-Ig-treated chimeras from NZW /BXSB mice, we enumerated the number of mutations in the FR regions and in CDRs of fulllength Vк5-43 sequences of GC B cells and plasma cells from the NZW/BXSB chimeras. No difference was observed in the frequency of mutations in GC cells (average 3.6 mutations per sequence in BAFF-R-Ig-treated versus 3.5 mutations per sequence in untreated chimeras, 26 and 28 sequences, respectively; Figure 6C) or plasma cells (average 2.4 versus 3.0 mutations per sequence, 15 and 16 sequences, respectively) or in the ratio of replacement to silent mutations in the CDR regions (Supplementary Table S4). The lightchain CDR1 of Vк5-43 makes an important contribution to antigen binding, since it consists of an asparagine-rich sequence QNISNN. In female NZW / BXSB chimeras that have attenuated autoreactivity, one or both of the two terminal asparagines of CDR1 is often replaced by serine or isoleucine. By contrast, replacement of these asparagines occurred rarely in either untreated or BAFF-R-Ig-treated male NZW /BXSB $3 \mathrm{H} 9$ chimeras (data not shown).

Both $V \kappa 5-43$ and $V \kappa 5-48$ light chains were sequenced from the GCs of NZB/W mice. There were fewer mutations per 

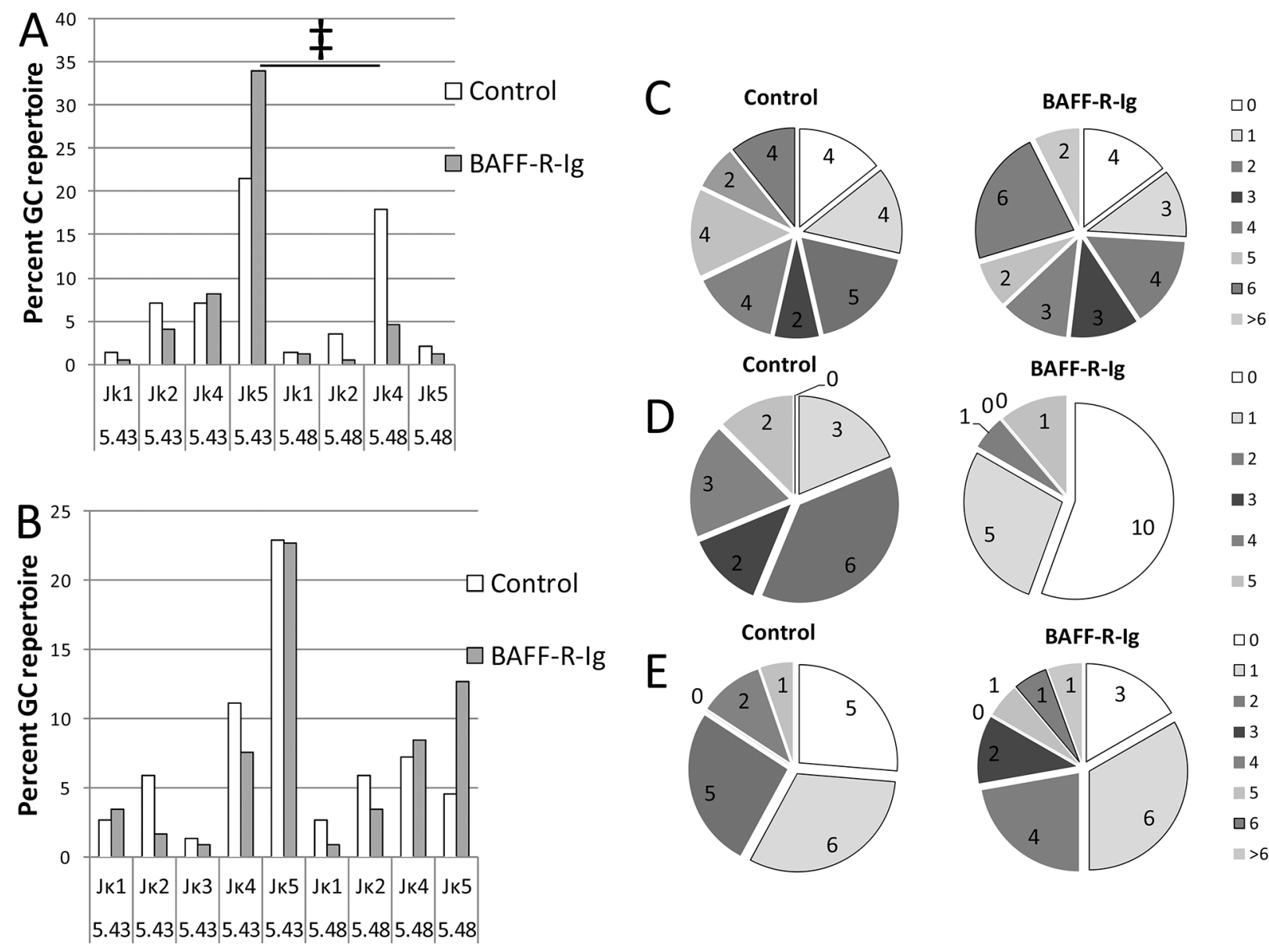

Figure 6. Distribution of $J_{\kappa}$ regions among $V_{\kappa} 5-45$ - and $V_{\kappa} 5-48$-encoded light chains as a percentage of total $G C$ light chains is shown in NZW/BXSB (A) and NZB/W (B) chimeras. The ratio of the two dominant light chains $V_{\kappa} 5-43 / 45 / J_{\kappa} 5$ and $V_{\kappa} 5-48 / J_{\kappa} 4$ is reversed in NZW/BXSB mice treated with BAFF-R-Ig $(\neq p<0.01)$. C-E show the mutation frequency per sequence for NZW/BXSB $V_{\kappa} 5-43$ (C), NZB/W $V_{\kappa} 5-43$ (D) and NZB/W VK5-48 (E).

sequence in $V \kappa 5-43$ light chains of BAFF-R-Ig-treated mice (6.3 versus 4.0 ) and fewer replacement mutations in the CDR regions of this light chain than in controls (Figure 6D, Supplementary Table S4, $p<0.01$ ). By contrast, there were more mutations per sequence in $V \kappa 5-48$ light chains of BAFF-R-Ig-treated NZB/W mice than controls (8.8 versus 4.7 ) but no difference in the number of replacement mutations in the CDR regions (Figure 6E, Supplementary Table S4). Interestingly, although the GC repertoire of both control and BAFF-R-Ig-treated NZB/W mice was dominated by $V \kappa 5-43$, these light chains were lost in the plasma cell repertoire of the BAFF-R-Ig-treated mice, which was dominated by $V \kappa 5-48$ light chains (Supplementary Figure S2).

\section{DISCUSSION}

In this study, we examined the regulation of 3H9-encoded anti-CL and anti-dsDNA autoantibodies by BAFF in two different mouse models of lupus. NZB/W female mice predominantly make an anti-dsDNA response, whereas NZW /BXSB male mice have an extra copy of TLR7 and make autoantibodies to chromatin, CL and RNA in a TLR7-dependent fashion $(20,32,33)$. We have shown in both strains that BAFF inhibition effectively delays the onset of SLE despite the continued generation of autoantibodies that deposit in the kidneys $(21,22)$. It is unknown whether BAFF inhibition alters the quality of the autoantibody response in SLE mice by altering either the naive or antigen-activated repertoires. It has been estimated that approximately $60 \%$ of 3H9-associated light chains are permissive for anti-dsDNA and/or anti-CL (but not RNA) autoreactivity when associated with the heavy chain (34), allowing us to analyze the effect of BAFF inhibition on the $3 \mathrm{H} 9$-associated light-chain repertoire. Our strategy of examining the autoreactive repertoire of single cells sorted from the various subsets avoided the potential of misinterpretation of the changes in 
the repertoire that could occur as a result of the substantial shifts in B-cell subsets that result from BAFF inhibition (21).

We show here, using a bone chimera system in which autoreactive B cells compete for survival with non-autoreactive B cells (20), that 3 H9 B cells were deleted centrally in both NZW/BXSB and $\mathrm{NZB} / \mathrm{W}$ strains and that this was not influenced by the availability of BAFF, consistent with the low expression of BAFF receptors by bone marrow $\mathrm{B}$ cells (35). Autoreactivity of the remaining $3 \mathrm{H} 9 \mathrm{~B}$ cells appeared to persist in the periphery, as manifested by downregulation of surface IgM on the mature naive transgenic cells. However, the degree of downregulation of the BCR was not affected by the availability of BAFF, nor did BAFF inhibition prevent autoreactive $3 \mathrm{H}^{+} \mathrm{B}$ cells from entering the GC or becoming plasma cells.

Follicular exclusion of anergic cells is an important precaution to limit the access of autoreactive B cells to the GC, where they can undergo class switching and somatic hypermutation that increase their self-reactivity $(36,37)$; excess BAFF permits follicular inclusion in several models (14). Therefore, it was intriguing that, in the $\mathrm{NZB} / \mathrm{W}$ strain, the naive repertoire of mice treated with the BAFF inhibitor no longer expressed the two $V \kappa$ genes $(V \kappa 3-4$ and $V \kappa 12-46)$ that dominate the marginal zone, suggesting that BAFF inhibition increases the stringency for follicular entry of B cells. The physiologic relevance of this finding is however unclear, since $V \kappa 3-4$ is not an autoreactive light chain when associated with $3 \mathrm{H} 9$ and $3 \mathrm{H} 9 / \mathrm{V} \kappa 12-46$ is not expressed in the GC or plasma cell repertoire of the NZW/BXSB strain.

In both NZW/BXSB and NZB/W mice, $3 \mathrm{H} 9 / \mathrm{V} \kappa 5$-encoded B cells are expanded in the GC and plasma cell repertoires and a large proportion of spontaneous IgG anti-CL and anti-dsDNA hybridomas from 3H9 transgenic NZW/BXSB and NZB/W mice use this $\mathrm{H} / \mathrm{L}$ pair (7). Importantly, although the autoreactive $V \kappa$ genes $V \kappa 5-43$ and $V \kappa 5-48$ were represented in the naive repertoire of NZB/W mice, their frequency was not decreased by BAFF inhibition. These findings in sum show that although there was substantial loss of naive B cells in BAFF-R-Ig-treated mice, with some skewing of the repertoire, the relevant autoreactive $3 \mathrm{H}^{+}$population did not appear to be preferentially targeted for deletion.

Although BAFF inhibition does not prevent the spontaneous formation of GCs or the generation of somatically mutated pathogenic IgG anti-dsDNA antibodies in lupus-prone mouse strains, the amount of tissue damage after the deposition of IgG autoantibodies in the kidneys is limited by BAFF inhibition (38). Whether this is due to a generalized decrease in inflammation that results from B-cell depletion or whether this is due to an alteration in the quality of the autoantibody response has not been determined.

The majority of 3H9-encoded GC and plasma cells from NZW/BXSB mice use $V \kappa 5-43, V \kappa 5-45$ and $V \kappa 5-48$ light chain genes $(7,20)$. When expressed in their germline configuration, $3 \mathrm{H} 9 / \mathrm{V} \kappa 5$-encoded antibodies bind to chromatin, but they bind weakly to dsDNA and CL (7). Furthermore, the J region influences the fine specificity of antibodies by using

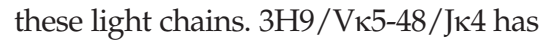
the highest affinity for CL and dsDNA, whereas $3 \mathrm{H} 9 / \mathrm{V} \kappa 5-43 / \mathrm{J} \kappa 5$ binds to chromatin and weakly to histone and dsDNA (7). By analyzing the autoreactive specificities of hybridomas generated from 3H9 transgenic NZW/BXSB mice, we showed that higher-affinity reactivity of 3H9/V5א-encoded autoantibodies toward CL and dsDNA is acquired by somatic mutation and that the two specificities can be acquired independently of each other, even when the same heavy- and light-chain genes are used (7). We have further shown that loss of one copy of TLR7 in male NZW/BXSB mice decreases the selection of B cells expressing the CL binding 3H9/ $\mathrm{V} \kappa 5-48 / \mathrm{Jk} 4$ both in the GCs and plasma cell compartment, whereas the 3H9/Vк5-43/45 $\mathrm{J} \kappa 5$ pair is found more frequently (20).

When we examined the GC B-cell repertoire of $3 \mathrm{H} 9$ bone marrow chimeras treated with BAFF-R-Ig, we found a difference between the two mouse strains. In NZW/BXSB mice, BAFF inhibition phenocopied the loss of one copy of TLR7 with a relative decrease of $V \kappa 5-48 / J \kappa 4$ and increase of $V \kappa 5-43 / 45 J \kappa 5$ chains in the GC and plasma cell compartments but no change in the frequency of somatic mutations in $V \kappa 5-43 / 45$ (20). BAFF and TLR7 share a common pathway in B cells, since TLR7-mediated signals upregulate B-cell expression of both TACI and BAFF-R, thereby enhancing B-cell survival $(39,40)$. In addition, signals through TACI cooperate with MyD88 in amplifying TLR-mediated signals (15). The changes that we observed in GC selection could in part be responsible for the decrease in maximal anti-CL titers reached in both TLR7-deficient and BAFF-R-Ig-treated NZW/BXSB mice. By contrast, the GC repertoire of NZB/W mice was similarly dominated by $\mathrm{V} \kappa 5$-encoded light chains but the relative distribution of $V \kappa 5-43$ and $V \kappa 5-48$ was not altered by BAFF inhibition. Instead, we found that the rate of somatic mutations was decreased in $V \kappa 5-43$-encoded light-chain genes from GC B cells, and these failed to be selected into the plasma cell repertoire. Nevertheless, anti-dsDNA titers were not altered by BAFF inhibition in this strain.

Our previous work in NZB/W lupus-prone mice expressing the autoreactive heavy-chain D42 showed that in the presence of competing non-autoreactive $B$ cells, deletion of autoreactive D42-expressing B cells occurs between the transitional type 1 and type 2 B-cell stage in the periphery and is further regulated by BAFF availability (3). This result did not prevent the selection of high-affinity germline-encoded autoreactive D42 B cells into the GC or plasma cell compartments $(3,41)$. Importantly, this dependence of autoreactive B cells on BAFF at the transitional B-cell stage does not apply uniformly to all autoreactive B cells. For example, B cells expressing the autoreactive HKI transgene are, like D42, deleted at the transitional to mature checkpoint, but when competition from non-autoreactive B cells is present, this deletion is not further enhanced by BAFF inhibition (42). These studies, together with the data that we present here, 
highlight the complexity of regulation of the autoreactive B-cell repertoire by BAFF.

\section{CONCLUSION}

Both the individual mouse strain and individual immunoglobulin specificity appear to dictate alterations in selection of the B-cell repertoire after BAFF inhibition. This may contribute to the heterogeneity of responses observed after BAFF inhibition in humans.

\section{ACKNOWLEDGMENTS}

This work was supported by the National Institutes of Health (NIAMS RO1 AR064811-01).

\section{DISCLOSURE}

The authors declare that they have no competing interests as defined by Molecular Medicine, or other interests that might be perceived to influence the results and discussion reported in this paper.

\section{REFERENCES}

1. Woods M, Davidson A, Zou YR. (2015) Defects in germinal center selection in SLE. Front. Immunol. 6:425.

2. Herlands RA, William J, Hershberg U, Shlomchik MJ. (2007) Anti-chromatin antibodies drive in vivo antigen-specific activation and somatic hypermutation of rheumatoid factor B cells at extrafollicular sites. Eur. J. Immunol. 37:3339-51.

3. Huang W, et al. (2011) BAFF/APRIL inhibition decreases selection of naive but not antigen-induced autoreactive B cells in murine systemic lupus erythematosus. J. Immunol. 187:6571-80.

4. Tipton CM, et al. (2015) Diversity, cellular origin and autoreactivity of antibody-secreting cell population expansions in acute systemic lupus erythematosus. Nat. Immunol. 16:755-65.

5. Cappione A 3rd, et al. (2005) Germinal center exclusion of autoreactive B cells is defective in human systemic lupus erythematosus. J. Clin. Invest. 115:3205-16.

6. Tiller T, et al. (2011) Development of self-reactive germinal center B cells and plasma cells in autoimmune Fc gammaRIIB-deficient mice. J. Exp. Med. 207:2767-78.

7. Moisini I, et al. (2012) The Yaa locus and IFN-alpha fine-tune germinal center B cell selection in murine systemic lupus erythematosus. J. Immunol. 189:4305-12.

8. Odegard JM, et al. (2008) ICOS-dependent extrafollicular helper T cells elicit IgG production via IL-21 in systemic autoimmunity. J. Exp. Med. 205:2873-86

9. Groom JR, et al. (2007) BAFF and MyD88 signals promote a lupuslike disease independent of T cells. J. Exp. Med. 204:1959-71.
10. Furie R, et al. (2011) A phase III, randomized, placebo-controlled study of belimumab, a monoclonal antibody that inhibits B lymphocyte stimulator, in patients with systemic lupus erythematosus. Arthritis Rheum. 63:3918-30.

11. Navarra SV, et al. (2011) Efficacy and safety of belimumab in patients with active systemic lupus erythematosus: a randomised, placebo-controlled, phase 3 trial. Lancet. 377:721-31.

12. Lesley $R$, et al. (2004) Reduced competitiveness of autoantigen-engaged $\mathrm{B}$ cells due to increased dependence on BAFF. Immunity. 20:441-53.

13. Thien M, et al. (2004) Excess BAFF rescues self-reactive B cells from peripheral deletion and allows them to enter forbidden follicular and marginal zone niches. Immunity. 20:785-98.

14. Liu Z, Davidson A. (2011) BAFF and selection of autoreactive B cells. Trends Immunol. 32:388-94.

15. He B, et al. (2010) The transmembrane activator TACI triggers immunoglobulin class switching by activating B cells through the adaptor MyD88. Nat. Immunol. 11:836-45.

16. Rahman ZS, Rao SP, Kalled SL, Manser T. (2003) Normal induction but attenuated progression of germinal center responses in BAFF and BAFF-R signaling-deficient mice. J. Exp. Med. 198:1157-69.

17. Ou X, Xu S, Lam KP. (2012) Deficiency in TNFRSF13B (TACI) expands T-follicular helper and germinal center B cells via increased ICOS-ligand expression but impairs plasma cell survival. Proc. Natl. Acad. Sci. U. S. A. 109:15401-6

18. Goenka R, et al. (2014) Local BLyS production by $\mathrm{T}$ follicular cells mediates retention of high affinity B cells during affinity maturation. J. Exp. Med. 211:45-56.

19. Scapini P, et al. (2010) Myeloid cells, BAFF, and IFN- $\gamma$ establish an inflammatory loop that exacerbates autoimmunity in Lyn-deficient mice. J. Exp. Med. 207:1757-73.

20. Boneparth A, et al. (2015) TLR7 influences germinal center selection in murine SLE. PLoS One. 10:e0119925.

21. Ramanujam M, et al. (2006) Similarities and differences between selective and nonselective BAFF blockade in murine SLE. J. Clin. Invest. 116:724-34.

22. Kahn P, et al. (2008) Prevention of murine antiphospholipid syndrome by BAFF blockade. Arthritis Rheum. 58:2824-34.

23. Ramanujam M, et al. (2009) Interferon-alpha treatment of female (NZW x BXSB)F(1) mice mimics some but not all features associated with the Yaa mutation. Arthritis Rheum. 60:1096-101.

24. Wang X, Huang W, Mihara M, Sinha J, Davidson A. (2002) Mechanism of action of combined short-term CTLA4Ig and anti-CD40 ligand in murine systemic lupus erythematosus. J. Immunol. 168:2046-53.

25. Akkerman A, et al. (2004) CTLA4Ig prevents initiation but not evolution of anti-phospholipid syndrome in NZW/BXSB mice. Autoimmunity. 37:445-51.

26. Ramanujam M, et al. (2004) Mechanism of action of transmembrane activator and calcium modulator ligand interactor-Ig in murine systemic lupus erythematosus. J. Immunol. 173:3524-34.

27. Liu Z, et al. (2011) Interferon-alpha accelerates murine systemic lupus erythematosus in a T cell-dependent manner. Arthritis Rheum. 63:219-29.

28. Wardemann H, et al. (2003) Predominant autoantibody production by early human B cell precursors. Science. 301:1374-7.

29. Lesser ML, Akerman MB. (2014) An exploratory graphical method for identifying associations in $\mathrm{r}$ x c contingency tables. J. Mod. Appl. Stat. Methods. 13:91-109.

30. Alabyev B, Rahman ZS, Manser T. (2007) Quantitatively reduced participation of anti-nuclear antigen B cells that down-regulate B cell receptor during primary development in the germinal center/memory B cell response to foreign antigen. J. Immunol. 178:5623-34.

31. Kirchenbaum GA, St Clair JB, Detanico T, Aviszus K, Wysocki LJ. (2014) Functionally responsive self-reactive B cells of low affinity express reduced levels of surface IgM. Eur. J. Immunol. 44:970-82.

32. Soni C, et al. (2014) B cell-intrinsic TLR7 signaling is essential for the development of spontaneous germinal centers. J. Immunol. 193:4400-14.

33. Jackson SW, et al. (2014) Opposing impact of B cell-intrinsic TLR7 and TLR9 signals on autoantibody repertoire and systemic inflammation. J. Immunol. 192:4525-32.

34. Ibrahim SM, Weigert M, Basu C, Erikson J, Radic MZ. (1995) Light chain contribution to specificity in anti-DNA antibodies. J. Immunol. 155:3223-33.

35. Mackay F, Schneider P. (2009) Cracking the BAFF code. Nat. Rev. Immunol. 9:491-502.

36. Brink R. (2014) The imperfect control of self-reactive germinal center B cells. Curr. Opin. Immunol. 28:97-101.

37. De Silva NS, Klein U. (2015) Dynamics of B cells in germinal centres. Nat. Rev. Immunol. 15:137-48.

38. Ramanujam M, et al. (2010) Selective blockade of BAFF for the prevention and treatment of systemic lupus erythematosus nephritis in NZM2410 mice. Arthritis Rheum. 62:1457-68.

39. Treml LS, et al. (2007) TLR stimulation modifies BLyS receptor expression in follicular and marginal zone B cells. J. Immunol. 178:7531-9.

40. Katsenelson N, et al. (2007) Synthetic CpG oligodeoxynucleotides augment BAFF- and APRIL-mediated immunoglobulin secretion. Eur. J. Immunol. 37:1785-95.

41. Eilat D, Wabl M. (2012) B cell tolerance and positive selection in lupus. J. Immunol. 189:503-9.

42. Nikbakht N, Migone TS, Ward CP, Manser T. (2011) Cellular competition independent of BAFF/B lymphocyte stimulator results in low frequency of an autoreactive clonotype in mature polyclonal B cell compartments. J. Immunol. 187:37-46.

Cite this article as: Boneparth A, et al. (2016) The Effect of BAFF inhibition on autoreactive B-cell selection in murine systemic lupus erythematosus. Mol. Med. 22:173-82. 